\title{
Gene family assignment-free comparative genomics
}

\author{
Daniel Doerr ${ }^{1,2^{*}}$, Annelyse Thévenin ${ }^{1,2}$, Jens Stoye ${ }^{1,2}$ \\ From Tenth Annual Research in Computational Molecular Biology (RECOMB) Satellite Workshop on Com- \\ parative Genomics \\ Niteroi, Brazil. 17-19 October 2012
}

\begin{abstract}
Background: The comparison of relative gene orders between two genomes offers deep insights into functional correlations of genes and the evolutionary relationships between the corresponding organisms. Methods for gene order analyses often require prior knowledge of homologies between all genes of the genomic dataset. Since such information is hard to obtain, it is common to predict homologous groups based on sequence similarity. These hypothetical groups of homologous genes are called gene families.
\end{abstract}

Results: This manuscript promotes a new branch of gene order studies in which prior assignment of gene families is not required. As a case study, we present a new similarity measure between pairs of genomes that is related to the breakpoint distance. We propose an exact and a heuristic algorithm for its computation. We evaluate our methods on a dataset comprising $12 \gamma$-proteobacteria from the literature.

Conclusions: In evaluating our algorithms, we show that the exact algorithm is suitable for computations on small genomes. Moreover, the results of our heuristic are close to those of the exact algorithm. In general, we demonstrate that gene order studies can be improved by direct, gene family assignment-free comparisons.

\section{Background}

In the field of comparative genomics, studying the relative order of genes in genomes is a popular practice to gain information about organisms and their relationships. This information ranges from transcription and functional linkage of genes such as correlated expression, the phylogeny of organisms, to detailed evolutionary dynamics of their genomes. Gene order methods are also incorporated in genome alignment strategies to identify regions that are subsequently used to anchor the alignment [1].

Genes are the atomic elements in gene order studies. Although no precise, formal definition is generally agreed upon, from the biological point of view a gene represents a specific inheritable entity in a particular locus on a chromosomal sequence in a particular organism. It often

\footnotetext{
* Correspondence: ddoerr@cebitec.uni-bielefeld.de

'Genome Informatics, Faculty of Technology, Center for Biotechnology (CeBiTec), Bielefeld University, Germany

Full list of author information is available at the end of the article
}

features a protein coding region. Nevertheless, the notion of a "gene" can also represent more fine-grained genetic structures such as protein domains or other functional elements of the genome.

Gene families. Many gene order studies hope for evolutionary relationships being resolved between all pairs of genes. Rested upon the biological concept of homology, such studies require information about orthology, paralogy and (potentially) xenology for each pair of genes in the dataset. This information is generally not given, hence it is common to cluster genes according to their sequence similarity. Sometimes such groups are called gene families, thus we will stick to this notion in the following.

Various databases exist, such as COG [2], eggNOG [3], Inparanoid [4], TreeFam [5], and OrthologID [6] (only to name a few) that offer gene family information. These databases can be divided into two groups: databases that primarily use sequence similarity to cluster genes into
C Biomed Central 
groups of co-orthologs; and tree-based databases offering reconstructed gene family trees [7].

The former group of databases provides usually more gene family data while covering a larger set of species. However, the contained information should always be taken with a pinch of salt: Even though high sequence similarity is a good indicator of homology, per se these gene families do not reflect an evolutionary relation. This is because they depend on arbitrary parameters of sequence comparison, similarity quantification, and clustering. Generally such parameters are user-controlled and influence the size and granularity of the computed gene families. Yet, the vast majority of these databases is uncurated or offers only a negligible amount of curated data.

Lacking a gene tree, within these gene families no differentiation can be made between in- and out-paralogs when comparing a specific pair of genomes. As is well-known, gene duplication and sub- or neofunctionalization occurs frequently in evolution. Hence the number of co-orthologous genes in a genome that are pooled into the same gene family grows the higher one ascends in the evolutionary tree. With increasing number of diverse genomes in the database, these gene families become less useful for gene order analyses, if only a close subset of taxa is of interest. The blemish of disregarding the evolutionary tree needed for truly resolving evolutionary relationships between genes of a given set of genomes is often covered by offering varying levels of granularity. This means that for some subtrees (but generally not for all) of the genomes in the database, gene families are recomputed with tighter parameters. Moreover, the computed sequencebased similarity estimates are rarely based on models of DNA evolution as these involve considerably more computational load. Subsequently differential evolutionary rates are disregarded, amplifying the dilemma of grouping genes based on sequence similarity: selecting too loose criteria in clustering genes to gene families may lead to the mistake that two genes are assigned to the same gene family while they are not homologous, whereas too strict criteria can split gene families although they should belong together [7].

Tree-based databases such as TreeFam and OrthologID may provide more accurate information desired for gene order studies. This is partly because the evolutionary relationships between genes in a gene family are considerd in more detail. Furthermore the species tree is taken into account while reconstructing the gene family trees. Also, tree-based databases tend to be more often manually curated than their sequence similarity based counterparts. In return, the provided gene family information is often sparse and covers not all genes of a genome. Moreover, such databases usually comprise only a handful of species. As a result, they are of limited use in gene order studies.
Gene content variations. Apart from model-free comparison or well-defined rearrangements in genomes, gene order studies can allow for additional biologically motivated operations of evolution. That is, genes can duplicate, emerge or become lost in the genome. Similarly, a gene family can grow or shrink, or new gene families can arise.

Gene order studies. Based on the concept of gene families, many gene order studies share a common data structure where chromosomes are represented as words drawn from a finite alphabet of gene families. The strength of this data structure lies in its simplicity; it allows to study the corresponding gene order problems in an abstract form composed of permutations or sequences over a set of characters. Another important advantage is the fact that homology is a binary and transitive relation. This led to the emergence of a multitude of efficient algorithms which solve gene order problems combinatorially.

In the following we will briefly review three different types of gene order studies. Dissimilarity measures such as the breakpoint distance [8] are used to calculate evolutionary distances between two or more genomes, without explicitly drawing on rearrangement operations. The breakpoint distance is defined by the number of unconserved adjacencies between characters of two genomes. For gene cluster detection, several competing models exist. One of them is based on the notion of approximate common intervals [9]. Thereby a gene cluster is defined as a set of maximal intervals, on two or more genomes, that share the same character set. Small differences between the set of characters constituting the gene cluster and the set of characters within the intervals are allowed. The number of tolerated differences as well as the minimal size of an interval is determined by a user-controlled parameter. Finally, a group of popular rearrangement models are based on the so-called double-cut-and-join (DCJ) operation $[10,11]$. By disrupting the genome on two different positions and rejoining the resulting ends, one aims to transform one genome into another by a minimal sequence of DCJ operations. This sequence is denoted sorting scenario.

Limits of the gene family concept. The concept of gene families comes with much benefit, but also has its detriments. On the one hand, gene family information can be gained with comparatively low effort by accessing various public databases or by direct computation. On the other hand, comparative studies based on uncurated gene families are hampered since data can be incorrect.

There are many reasons why the exclusive, binary membership relation between genes and gene families is disputable in itself. For one, most gene families are uncurated, hence it would be supporting in constitutive analyses to distinguish between weak and strong assumptions of homology between genes in supporting their membership 
to one or more gene families. Moreoever, the gene family concept disregards the facts that gene families may share conserved protein domains and that genes may fuse with others in the course of evolution.

In this paper we promote the idea that gene order studies can be performed without prior gene family assignment. We propose direct use of similarity values because such information not only allows to make more substantiated choices in resolving gene order in subsequent analyses, but can sometimes better reflect the biological reality. In support of our case, we present a new approach to calculate the number of conserved adjacencies, which is a similarity measure related to the breakpoint distance, without the use of gene families. Our method is based on a weighted bipartite graph, representing pairwise similarities between genes of two genomes. We show that this allows for stable adjacency analyses when similarities are calculated based on sequence similarity.

In the "Methods" section we will introduce the problem setting formally and devise an exact algorithm as well as a heuristic for its solution. In the "Experiments and Discussion" section we discuss the performance of our presented method on this dataset and compare results with former work. The manuscript closes with concluding remarks and future prospects in the "Conclusions" section.

\section{Methods}

\section{Formal problem description}

Genome model. Let $\mathcal{G}$ be the universe of all genes, then a chromosome is defined as a sequence of genes $\left(^{\circ}, g_{1}\right.$, $\left.g_{2}, \ldots, g_{n-1},{ }^{\circ}\right)$, with $g_{i} \in \mathcal{G}$ for all $i=1, \ldots, n-1$, flanked by telomeric ends represented by "o". Depending on the type of gene order study, chromosomes can be signed or unsigned. If signed, a gene $g$ has a direction indicated by $-g$ or $+g$ (but it is common to omit the " + "), which represents the relative orientation of each gene along the chromosome. A chromosome can also be circular as it is often observed in bacteria; in this case, it does not exhibit telomeric ends, implying that the outermost genes adjoin. For the time being, let us assume that a genome is unichromosomal and linear, since the general case of our model can be easily inferred. The size of a genome $G$ with $n-1$ genes is $|G|=n$. In order to refer to the $i$ th gene of $G$, we use the notation $G[i]$. Further, let $\sigma: \mathcal{G} \times \mathcal{G} \rightarrow[0,1]$ be a normalized similarity measure between all pairs of genes.

Graph representation. Given two genomes $G_{1}, G_{2}$ of lengths $n_{1}=\left|G_{1}\right|$ and $n_{2}=\left|G_{2}\right|$, respectively. We define an ordered weighted bipartite graph $B=\left(G_{1}, G_{2}, E\right)$ over both genomes in which the order is given by the chromosomal order of genes (see example in Figure 1(a)). For $0<i<n_{1}$, $0<k<n_{2}$, a pair of genes, one from $G_{1}$ and one from $G_{2}$, is connected by an edge $e_{i k}:=\left(G_{1}[i], G_{2}[k]\right) \in E$ with edge weight $w\left(e_{i k}\right):=\sigma\left(G_{1}[i], G_{2}[k]\right)$ if and only if $\sigma\left(G_{1}[i]\right.$,
$\left.G_{2}[k]\right)>0$. Telomeres are always connected with edges of weight 1: $w\left(e_{00}\right)=w\left(e_{0 n_{2}}\right)=w\left(e_{n_{1} 0}\right)=w\left(e_{n_{1} n_{2}}\right)=1$, as depicted in our example in Figure 1(a). We call a gene $g_{i}$ $\in G_{x}, x \in\{1,2\}$ unconnected if there exists no gene $g_{k} \in$ $G_{y}, y \in(\{1,2\} \backslash\{x\})$ such that $\sigma\left(g_{\dot{v}} g_{k}\right)>0$.

Unconnected genes are omitted from the chromosomal sequences. The remaining genes form connected components of size two or larger. Let $\mathcal{C}$ denote the set of all such connected components of $B$, then for some $C \in \mathcal{C}$ and $x \in\{1,2\}, C_{x}$ denotes the set of all genes of $C$ that are part of $G_{x}$. Given $B$, we will be interested in finding a set of disjoint edges. Such a set, denoted by $\mathcal{M}$, is known as matching.

Matchings. Let us assume for now that a matching $\mathcal{M}$ between $G_{1}$ and $G_{2}$ is given. \#edg $(\mathcal{M})$ denotes the number of edges in $\mathcal{M}$. We call a gene saturated if it is incident to an edge of the matching. A pair of genes $\left(G_{x}\right.$ $\left.[i], G_{x}[j]\right)$, with $x \in\{1,2\}$ and $0 \leq i<j \leq n_{x}$, is a consecutive pair if no saturated gene lies between them.

Recall that genes have directions; the orientation of a gene $g$ is determined by the following function:

$$
\operatorname{sgn}(g)= \begin{cases}1 & \text { if } g>0 \\ -1 & \text { if } g<0 \\ 0 & \text { if } g \text { is a telomere }\end{cases}
$$

Two consecutive pairs of genes $\left(G_{1}[i], G_{1}[j]\right)$ and $\left(G_{2}\right.$ [k], $\left.G_{2}[\ell]\right)$, with $0 \leq i<j \leq n_{1}$ and $0 \leq k, \ell \leq n_{2}$, form a conserved adjacency if the corresponding edges $e_{i k}, e_{j \ell}$ are part of $\mathcal{M}$ and:

$$
\begin{aligned}
& \text { 1. for } k<\ell, \operatorname{sgn}\left(G_{1}[i]\right)=\operatorname{sgn}\left(G_{2}[k]\right) \text { and } \operatorname{sgn}\left(G_{1}[j]\right)= \\
& \operatorname{sgn}\left(G_{2}[\ell]\right) \text { or } \\
& \text { 2. for } k>\ell, \operatorname{sgn}\left(G_{1}[i]\right) \neq \operatorname{sgn}\left(G_{2}[k]\right) \text { and } \operatorname{sgn}\left(G_{1}[j]\right) \neq \\
& \operatorname{sgn}\left(G_{2}[\ell]\right) \text {. }
\end{aligned}
$$

For example, in Figure 1(b) the consecutive gene pairs $(2,-3)$ and $(6,-7)$ represent a conserved adjacency. Telomeres located at the first and last position of the chromosomes are "unsigned" and thus can be used to form adjacencies in both directions. We denote the sum of all conserved adjacencies in a matching $\mathcal{M}$ by $\#$ adj $(\mathcal{M})$.

Among all possible matchings between $G_{1}$ and $G_{2}$, we search the biologically most relevant. A well-known matching is the maximal weighted matching, which maximizes the sum of weights of disjoint edges of a bipartite graph. In our example, Figure 1(b) represents a maximal weighted matching. This kind of matching can be motivated from a biological point of view: The higher the sequence similarity between two genes, the more likely they are homologs. Yet, if we want to construct a biologically meaningful matching, we must not only consider edge weights, but also the ability of two edges forming a conserved adjacency in the final matching. We somehow 


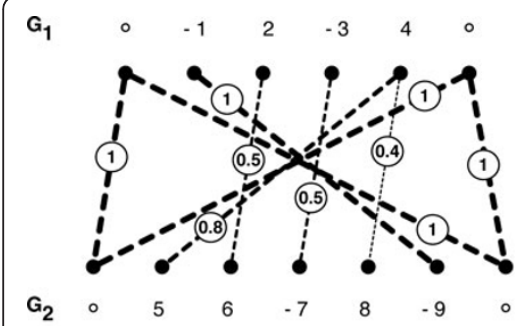

(a)

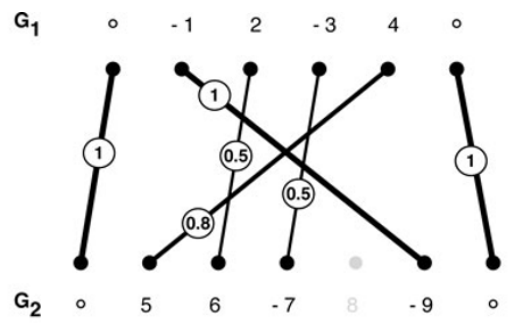

(b)

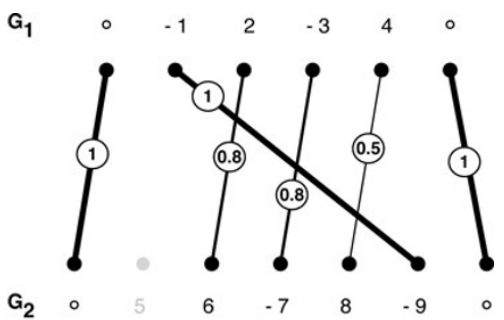

(c)

Figure 1 Bipartite graph and matching. (a) The ordered weighted bipartite graph represents similarity relationships between genes of two genomes. (b) and (c) represent solutions to Problem 1 with $\alpha \rightarrow 0$ and $\alpha=1$ respectively.

want to maximize for the number of conserved adjacencies in the final matching, because we observe from biological data that rearrangements of genes in genomes occur parsimoniously. However, we want to prevent that conserved adjacencies incorporating low-weight edge pairs are formed if the corresponding genes are incident to higherweight edges (see Figure 1(c)). Consequently we propose the following scoring scheme for conserved adjacencies:

$$
s(i, j, k, l)= \begin{cases}w\left(e_{i k}\right) \cdot w\left(e_{j l}\right) & \text { if }\left(G_{1}[i], G_{1}[j]\right) \text { and }\left(G_{2}[k], G_{2}[\ell]\right) \text { form a conserved adjacency } \\ 0 & \text { otherwise }\end{cases}
$$

In our matching we want to promote conserved adjacencies but also edges: Because in the presented approach, connected components are larger than gene families, we aim to match more than one pair per connected component, even in the case they do not exhibit adjacencies. Hence we quantify the quality of a matching $\mathcal{M}$ according to the following functions, where $i, j$ indicate indices in genome $G_{1} ; k, \ell$ in $G_{2}$ :

$$
\begin{aligned}
& \operatorname{adj}(\mathcal{M})=\sum_{\substack{0 \leq i<j \leq\left|G_{1}\right|, 0 \leq k, l \leq\left|G_{2}\right|}} s(i, j, k, l) \\
& \operatorname{edg}(\mathcal{M})=\sum_{e \in \mathcal{M}} w(e)^{2}
\end{aligned}
$$

Notice that the edge weights in the sum of the Equation 3 are squared to match the dimension of Equation 2. Optimizing a matching with respect to $e d g(\mathcal{M})$ will result in a maximal weighted matching in the graph model we introduced above. As our overall objective function we propose a linear combination between Equations 2 and 3. We allow the user to balance between those two quantities by a parameter $\alpha$. Moreover it is reasonable to add the constraint that at least one edge per connected component of the bipartite graph between $G_{1}$ and $G_{2}$ must be contained in the matching; The matching obtained is an intermediate matching.

Problem 1 (Family-free(FF)-Adjacencies) Given two genomes $G_{1}$ and $G_{2}$, a normalized similarity measure $\sigma$, and some $\alpha L] 0,1]$, find a matching $\mathcal{M i n} B=\left(G_{1}, G_{2}, E\right)$ such that at least one edge per connected component of $B$ is contained in Mand the following formula is maximized:

$$
\mathcal{F}_{\alpha}(\mathcal{M})=\alpha \cdot \operatorname{adj}(\mathcal{M})+(1-\alpha) \cdot \operatorname{edg}(\mathcal{M}) .
$$

Problem FF-Adjacencies can be reduced to two problems that were addressed already by Tang and Moret [12] and Angibaud et al. [13]. Therefore, let us consider equivalent conditions that prevail if gene families are given: In the bipartite graph $B=\left(G_{1}, G_{2}, E\right)$ between two genomes $G_{1}$ and $G_{2}$ all edges have edge weight 1 and all connected components are cliques. Then finding a solution to Problem FF-Adjacencies with $\alpha=1$ is equivalent to finding a matching that maximizes the number of adjacencies between two genomes with duplicate genes under the intermediate model [13]. If $\alpha$ comes close enough to 0 , we will obtain a maximum matching, yet maximizing the number of adjacencies [12]. The case where family conditions are met also reveals the difference between an arbitrary maximum matching and the maximum matching found by solving Problem FF-Adjacencies for $\alpha \rightarrow 0$.

The reduced problems presented above being already NP-hard, the problem FF-Adjacencies is NP-hard as well. In the next two subsections we propose first an exact algorithm, FFAdj-Int, to solve Problem FF-Adjacencies and then a fast heuristic approach.

\section{Exact algorithm}

Our algorithm FFAdj-Int solving Problem FF-Adjacencies is based on previous work in [13]. The idea is to translate the problem into a 0-1 linear program. That means we define a set of constraints (linear inequations) whose variables are booleans and an objective function (maximization or minimization of a linear formula). Then, we use a solver to assign a value for each variable such that the constraints are verified and the objective is optimized.

The program FFAdj-Int considers two linear genomes $G_{1}$ and $G_{2}$ of respective lengths $n_{1}$ and $n_{2}$, a number 
$\alpha \in] 0,1]$, and a function $\sigma: \mathcal{G} \times \mathcal{G} \rightarrow[0,1]$. The objective, the variables and the constraints are defined in Figure 2 and are briefly discussed hereafter.

\section{Variables:}

- Variables $a(i, k), 0 \leq i \leq n_{1}$ and $0 \leq k \leq n_{2}$, define a matching $\mathcal{M}: a_{i, k}=1$ if and only if the gene at position $i$ in $G_{1}$ is matched with the gene at position $k$ in $G_{2}$ in $\mathcal{M}$, i.e. $e_{i k} \in \mathcal{M}$.
- Variables $b_{x}(i), x \in\{1,2\}$ and $0 \leq i \leq n_{x}$, represent the genes saturated by $\mathcal{M}: b_{x}(i)=1$ if and only if the gene at position $i$ in $G_{x}$ is saturated by the matching $\mathcal{M}$. Clearly, $\Sigma_{0} \leq i \leq n 1 b_{1}(i)=\Sigma_{0} \leq k \leq n 2$ $b_{2}(k)$, and this is precisely the size of the matching $\mathcal{M}$.

- Variables $c_{x}(i, j), x \in\{1,2\}$ and $0 \leq i<j \leq n_{x}$, represent consecutive pairs according to the matching $\mathcal{M}: c_{x}(i, j)=1$ if and only if the genes at

Program FFAdj-Int

\section{Objective :}

Maximize

$$
\alpha \cdot \sum_{0 \leq i<j \leq n_{1}} \sum_{0 \leq k, l \leq n_{2}} s(i, j, k, \ell) \cdot d(i, j, k, \ell)+(1-\alpha) \cdot \sum_{\substack{0 \leq i \leq n_{1} \\ 0 \leq k \leq n_{2}}} \sigma\left(G_{1}[i], G_{2}[k]\right)^{2} \cdot a(i, k)
$$

\section{Constraints :}

(C.01) $\forall 0 \leq i \leq n_{1}, \sum_{0 \leq k \leq n_{2}} a(i, k)=b_{1}(i)$

$$
\forall 0 \leq k \leq n_{2}, \sum_{0 \leq i \leq n_{1}} a(i, k)=b_{2}(k)
$$

(C. 02) $\forall x \in\{1,2\}, \forall C \in \mathcal{C}, \sum_{i \in C_{x}} b_{x}(i) \geq 1$

(C.03) $\forall x \in\{1,2\}, \forall 0 \leq i<j \leq n_{x}, c_{x}(i, j)+\sum_{i<p<j} b_{x}(p) \geq 1$

(C. 04) $\forall x \in\{1,2\}, \forall 0 \leq i<p<j \leq n_{x}, c_{x}(i, j)+b_{x}(p) \leq 1$

(C.05) $\forall 0 \leq i<j \leq n_{1}, \forall 0 \leq k, \ell \leq n_{2}, \forall k \neq \ell, \sigma\left(G_{1}[i], G_{2}[k]\right)>0, \sigma\left(G_{1}[j], G_{2}[l]\right)>0$ such that $\left(k<\ell\right.$ and $\operatorname{sgn}\left(G_{1}[i]\right)=\operatorname{sgn}\left(G_{2}[k]\right)$ and $\left.\operatorname{sgn}\left(G_{1}[j]\right)=\operatorname{sgn}\left(G_{2}[\ell]\right)\right)$, or $\left(k>\ell\right.$ and $\operatorname{sgn}\left(G_{1}[i]\right)=-\operatorname{sgn}\left(G_{2}[k]\right)$ and $\left.\operatorname{sgn}\left(G_{1}[j]\right)=-\operatorname{sgn}\left(G_{2}[\ell]\right)\right)$, $a(i, k)+a(j, \ell)+c_{1}(i, j)+c_{2}(k, \ell)-d(0 i, j, k, \ell) \leq 3$ $a(i, k)-d(i, j, k, \ell) \geq 0$ $a(j, \ell)-d(i, j, k, \ell) \geq 0$ $c_{1}(i, j)-d(i, j, k, \ell) \geq 0$ $c_{2}(k, \ell)-d(i, j, k, \ell) \geq 0$

(C.06) $\forall 0 \leq i<j \leq n_{1}, \forall 0 \leq k, \ell \leq n_{2}$ such that $k=\ell$,

$$
\begin{aligned}
& \text { or } \sigma\left(G_{1}[i], G_{2}[k]\right)=0, \\
& \text { or } \sigma\left(G_{1}[j], G_{2}[\ell]\right)=0, \\
& \text { or }\left(k<\ell \text { and }\left(\operatorname{sgn}\left(G_{1}[i]\right) \neq \operatorname{sgn}\left(G_{2}[k]\right) \text { or } \operatorname{sgn}\left(G_{1}[j]\right) \neq \operatorname{sgn}\left(G_{2}[\ell]\right)\right)\right), \\
& \text { or }\left(k>\ell \text { and }\left(\operatorname{sgn}\left(G_{1}[i]\right) \neq-\operatorname{sgn}\left(G_{2}[k]\right) \text { or } \operatorname{sgn}\left(G_{1}[j]\right) \neq-\operatorname{sgn}\left(G_{2}[\ell]\right)\right)\right), \\
& d(i, j, k, \ell)=0
\end{aligned}
$$

\section{Domains :}

$$
\begin{gathered}
\forall x \in\{1,2\}, \forall 0 \leq i<j \leq n_{1}, \forall 0 \leq k, \ell \leq n_{2}, k \neq \ell \\
a(i, k), b_{x}(i), c_{x}(i, j), d(i, j, k, \ell) \in\{0,1\}
\end{gathered}
$$

Figure 2 Program FFAdj-Int. Program FFAdj-Int for finding an intermediate matching that maximizes the objective $\left.\left.\mathcal{F}_{\alpha}(\alpha \in] 0,1\right]\right)$ between two genomes $G_{1}$ and $G_{2}$. 
positions $i, j$ in $G_{x}$ are saturated by $\mathcal{M}$ and no gene at position $p, i<p<j$, is saturated by $\mathcal{M}$.

- Variables $d(i, j, k, e), 0 \leq i<j \leq n_{1}, 0 \leq k, \ell \leq n_{2}$, represent conserved adjacencies according to the matching $\mathcal{M}: d(i, j, k, e)=1$ if and only if $s(i, j, k, \ell)>0$.

Because the matching is possible only between similar genes, the variables $a(i, k)$ and $d(i, j, k, \ell)$ are not defined whenever $\sigma\left(G_{1}[i], G_{2}[k]\right)=0$. Similarly, the variables $d(i, j, k, \ell)$ are not defined if $\sigma\left(G_{1}[j], G_{2}[\ell]\right)=0$.

\section{Objective:}

The goal of FFAdj-Int is to find a matching $\mathcal{M}$ between the two considered genomes that maximizes the formula $\left.\left.\mathcal{F}_{\alpha}(\alpha \in] 0,1\right]\right)$. Hence, the objective of FFAdj-Int reduces to maximizing the sum of all variables $d(i, j, k, \ell)$ multiplied by $\alpha \cdot s(i, j, k, \ell)$, plus the sum of all variables $a(i, k)$ multiplied by $(1-\alpha) \cdot \sigma(i, k)^{2}$

\section{Constraints:}

Assume $x \in\{1,2\}, 0 \leq i<j \leq n_{1}$ and $0 \leq k, \ell \leq n_{2}$.

- Constraints in (C.01) ensure that each gene of $G_{1}$ and of $G_{2}$ is saturated at most once, i.e. $b_{1}(i)=1$ (resp. $b_{2}(k)=1$ ) if and only if there exists a unique $k$ (resp. $i$ ) such that $a(i, k)=1$, i.e. $e_{i k} \in \mathcal{M}$.

- Constraints in (C.02) ensure that the matching $\mathcal{M}$ is an intermediate matching, we want for each component at least one edge in the matching $\mathcal{M}$. For each component $C \in \mathcal{C}$, the sum of the variables $b_{x}$ (i) for $i \in C_{x}$ must be greater than or equal to 1 .

- Constraints in (C.03) and (C.04) express the definition of consecutive pairs, thus fixing the values of the variables $c_{x}$. The variable $c_{x}(i, j)\left(0 \leq i<j \leq n_{x}\right)$ is equal to 1 if and only if there exists no $p$ such that $I<p<j$ and $b_{x}(p)=1$. It is worth noticing that the constraints do not force the variables $c_{x}(i, j)$ to have exactly the values we intuitively wish according to the above mentioned interpretation. Here, we accept that $c_{x}(i, j)=1$ even if the gene at position $i$ or $j$ is not saturated. However, this will pose no problem in the sequel.

- Constraints in (C.05) and (C.06) define variables $d$. Knowing the variables $d(i, j, k, \ell)$ are defined only if $\sigma(i, k)>0$ and $\sigma(j, \ell)>0$, constraints $(\mathbf{C . 0 5})$ and (C.06) ensure that we have $d(i, j, k, \ell)=1$ if and only if all variables $a(i, k), a(j, e), c_{1}(i, j)$ and $c_{2}(k, e)$ are equal to 1 and the signs and the order of $G_{1}[i]$, $G_{1}[j], G_{2}[k]$ and $G_{2}[\ell]$ are consistent with the definition of conserved adjacencies.

The program FFAdj-Int has $O\left(\left(n_{1} n_{2}\right)^{2}\right)$ constraints and $O\left(\left(n_{1} n_{2}\right)^{2}\right)$ variables, which could result in a timeconsuming computation.
So far we have used only one simple rule in order to reduce the space complexity: By the definition of the intermediate model, for all components with only two genes, $G_{1}[i]$ and $G_{2}[k]$, the edge $e_{i k}$ is in $\mathcal{M}$. By the constraints (C.01) and (C.03), we already enforce that the variables $a(i, k), b_{1}(i)$ and $b_{2}(k)$ are equal to 1 . The rule is based on the fact that there is no possible consecutivity in $\mathcal{M}$ between $G_{1}[s]$ and $G_{1}[t]$ (resp. $G_{2}[s]$ and $G_{2}[t]$ ) such that $0 \leq s<i<t \leq n_{1}$ (resp. $0 \leq s<k<t \leq n_{2}$ ), i.e. $c_{1}$ $(s, t)$ (resp. $c_{2}(s, t)$ ) is equal to 0 . The corresponding variables $d(s, t, .,).($ resp. $d(., ., s, t)$ and $d(., ., t, s))$ are also equal to 0 .

\section{Heuristic}

Because of the combinatorial explosion, FFAdj-Int does not solve Problem FF-Adjacencies for all pairs of complete, larger genomes. But, we will see in the "Experiments and discussion" section that FFAdj-Int allows to obtain enough results to evaluate our heuristic presented in this section. It is based on similar ideas as the heuristic IILCS in [13]. IILCS allows to compute the number of adjacencies between two genomes when gene families are known, under three models: exemplar (only one match by gene family), intermediate, and maximum. IILCS resolves our Problem FF-Adjacencies in the particular case where $\alpha=$ 1 and each component represents a gene family, i.e. each component is a clique where the weight of each edge is 1 .

The heuristic IILCS is a greedy algorithm based on the notion of $L C S$, Longest Common Substring: Given two genomes $G_{1}$ and $G_{2}$, an $L C S$ is a longest string $S$ such that $S$ is a (consecutive) substring in $G_{1}$ and $G_{2}$, up to a complete reversal (opposite sign and reverse order). The idea is to match, at each iteration, all the genes that are in an LCS. If there are several LCSs, one is chosen arbitrarily. At each iteration, not only we match an $L C S$, but we also remove each unmatched gene from the genome, for which there is no unmatched gene of same component in the other genome. The process (determination of LCS, match and deletion of genes) is iterated until a satisfying matching is obtained. Under the intermediate model, the iteration is stopped when there is at least one edge in $\mathcal{M}$ for each component.

For the problem FF-Adjacencies,we update the IILCS heuristic by three modifications. The goal of the first change is to take into account our objective in the choice of common substrings. In each iteration we match the common substring that maximizes locally $\left.\left.\mathcal{F}_{\alpha}(\alpha \in] 0,1\right]\right)$, i.e. the sum of weights of adjacencies and edges. We call this common substring a Maximum Common Substring (MCS). The second modification is an improvement that may also be applied to the original IILCS heuristic: After the deletion of an unsaturated gene $g_{1}$, such that there is no unmatched gene $g_{2}$ with $\sigma\left(g_{1}, g_{2}\right)>0$, we attempt to 
increase the size of each previously matched MCS by extending it on both extremities. The next and the last change is related to the model. We have two options to increase our objective. The first one is to stop the iteration only when we have at least one edge per component and when the size of the MCS of the current iteration is below 2 . In the case of the gene family constraints, this criterion improves also the results of IILCS. The second possibility is to stop the iteration only when there is no more edge between unmatched genes. In comparison to the first possibility, we increase our objective $\mathcal{F}_{\alpha}(\alpha \in]$ $0,1]$ ) only if $\alpha \neq 1$, so not in the context of IILCS. We choose this second possibility because the objective is bigger, but it is important to understand that then we also increase the number of breakpoints. We call this heuristic FFAdj-MCS.

\section{Experiments and Discussion Data}

To evaluate our algorithms, we chose $12 \gamma$-proteobacteria genomes from the dataset of Lerat et al. that was also used in previous work and which is to some degree considered as a standard reference dataset in comparative genomics [13-15]. The suggested phylogeny has been confirmed in later studies e.g. Williams et al. [16]. The genomic data including gene annotations have been obtained from NCBI under the accession numbers listed in Table 1.

All genomes comprise a single, circular chromosome. In support of simplified code but at the expense of accuracy, our implemented algorithms do not allow a chromosome to be circular, even though this is permitted by our presented model. However, the maximal error made by this inaccuracy in comparing two genomes is at most one adjacency, which is negligible in our analysis. The genomes were linearized in the order inherent to the NCBI data, and telomeres were added at the beginning and at the end of the resulting chromosomal sequences.
Pairwise normalized similarities were obtained using the relative reciprocal BLAST score (RRBS) [17]. Genes were compared on the basis of their encoding protein sequence using BLASTP with an e-value threshold of 0.1 , disabled query sequence filtering, and disabled composition-based score adjustments. All computations were performed on a computer system with 32 gigabytes of main memory.

\section{Exact Algorithm vs Heuristic}

Using the CPLEX solver we ran the 0-1 linear programs obtained by FFAdj-Int for 66 pairs of genomes with varying values of $\alpha(\alpha \in\{0.001,0.3,0.5,0.8,1\})$. A detailed table of results is enclosed in Additional file 1. In some cases, in particular larger and close genomes, we were not able to obtain results due to the lack of sufficient memory; We obtained results for 43 (resp. 63, 61, 54 and 48) pairs of genomes for $\alpha=0.001$ (resp. 0.3, 0.5, 0.8 and 1). For 40 pairs of genomes we could solve the 0-1 linear program for all values of $\alpha$. These results are summarized in Figure 3 , showing mean values of $\mathcal{F}_{\alpha}(\mathcal{M}), \operatorname{adj}(\mathcal{M})$, and $\operatorname{edg}(\mathcal{M})$ plotted as a function of $\alpha$. The same plot also includes results of our heuristic showing similar trends. Both, adj $(\mathcal{M})$, and $\operatorname{edg}(\mathcal{M})$ show little change while varying $\alpha$. This indicates that the set of high-scoring adjacencies and highweight edges, that contribute the most, are largely shared among the matchings with different $\alpha$. The abrupt drop in the mean value of $e d g(\mathcal{M})$ for $\alpha=1$ results from the fact that for this value the second term of Equation 4 drops out. Consequently, single pairs (i.e. those that do not share conserved adjacencies) of matched genes $\left(G_{1}[i], G_{2}[k]\right)$ are removed from the genomes during the resolution of the linear program. On the other side, because the heuristic iteratively constructs a matching until full saturation, the value for $\operatorname{edg}(\mathcal{M})$ for $\alpha=1$ remains high.

In our evaluation of FFAdj-MCS, we demonstrate that it represents a feasible heuristic for Problem FF-Adjacencies. In Table 2 the relative deviation of the heuristic results

Table 1 Genomic dataset.

\begin{tabular}{|c|c|c|c|c|}
\hline Species/strain name & Short name & Accession No. & Size (bp) & \#Genes \\
\hline Buchnera aphidicola APS & BAPHI & NC_002528 & 640681 & 564 \\
\hline Escherichia coli K12 & ECOLI & NC_000913 & 4639675 & 4320 \\
\hline Haemophilus influenzae Rd & HAEIN & NC_000907 & 1830138 & 1657 \\
\hline Pseudomonas aeruginosa PA01 & PAERU & NC_002516 & 6264404 & 5571 \\
\hline Pasteurella multocida Pm70 & PMULT & NC_002663 & 2257487 & 2012 \\
\hline Salmonella typhimurium LT2 & SALTY & NC_003197 & 4857432 & 4423 \\
\hline Wigglesworthia glossinidia brevipalpis & WGLOS & NC_004344 & 697724 & 611 \\
\hline Xanthomonas axonopodis pv. citri 306 & XAXON & NC_003919 & 5175554 & 4312 \\
\hline Xanthomonas campestris & XCAMP & NC_003902 & 5076188 & 4179 \\
\hline Xylella fastidiosa $9 \mathrm{a} 5 \mathrm{c}$ & XFAST & NC_002488 & 2679306 & 2766 \\
\hline Yersinia pestis CO_92 & YPEST-CO92 & NC_003143 & 4653728 & 3885 \\
\hline Yersinia pestis KIM5 P12 & YPEST-KIM & NC_004088 & 4600755 & 4048 \\
\hline
\end{tabular}

The genomic dataset of our analysis comprises $12 \gamma$-proteobacteria from Lerat et al. [14]. 


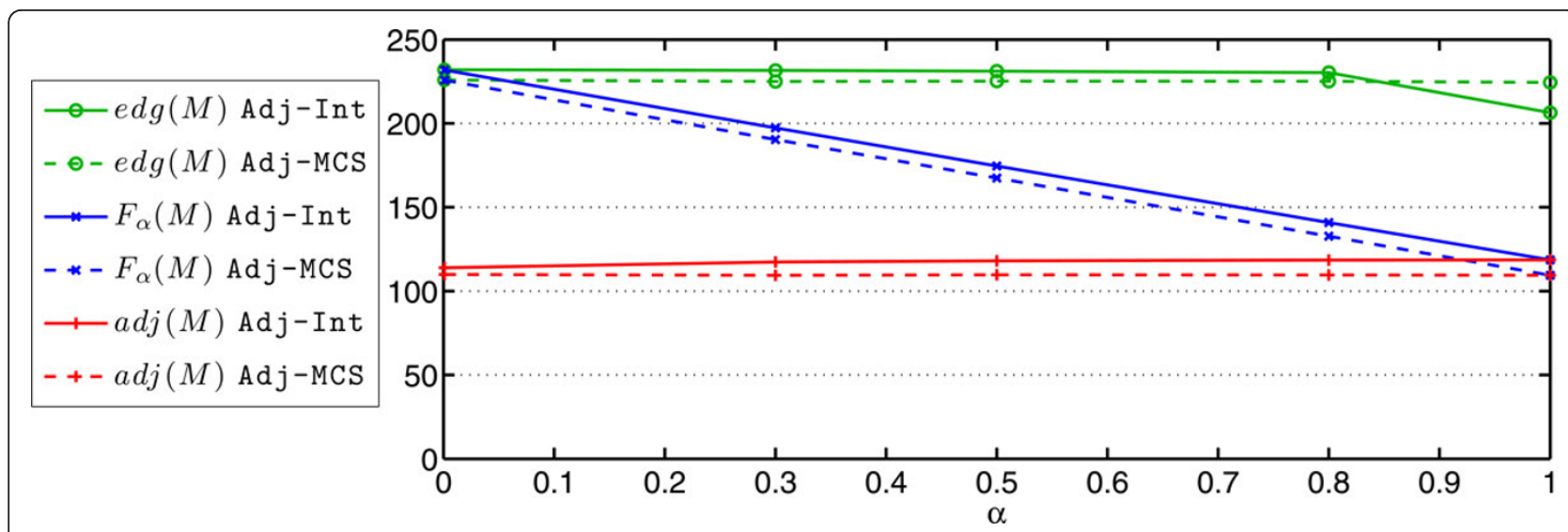

Figure 3 Solving FF-Adjacencies for various values of $\alpha$. Mean values of $\mathcal{F}_{\alpha}(\mathcal{M})$, $\operatorname{adj}(\mathcal{M})$ and edg( $\left.\mathcal{M}\right)$ plotted as a function of $\alpha$ for 40 genomes based on results of FFAdj-Int (solid lines) and FFAdj-MCS (dashed lines).

from the solutions of FFAdj-Int are listed. In the worst case, where $\alpha=1$, the heuristic deviates in the objective by less than $10 \%$. Due to its greedy nature, in all cases but one the size of the matching is larger than in the optimal solution. In order to evaluate the gain of the family-free analysis, we compare the results of FFAdj-Int against those of Adjacencies-Intermediate-Matching [13]. The linear program Adjacencies-Intermediate-Matching maximizes the number of adjacencies under the intermediate model between two genomes with gene family constraints. To compare the number of adjacencies (a common measure of these two programs) correctly, we must take into account two facts. First, the number of genes of the studied genomes differ. In [13], the authors used gene annotations and gene families that are reported in [15] whereas in our current study we employed gene annotations from NCBI. Nevertheless, the difference in number of genes is on average $0.02 \%$ per genome. Secondly, the genes for Adjacencies-Intermediate-Matching are unsigned, which artificially increases the number of adjacencies. We observed many more adjacencies in the results of FFAdjInt and of FFAdj-MCS than in Adjacencies-Intermediate-Matching. Furthermore, the matching produced by

Table 2 Exact algorithm vs heuristic.

\begin{tabular}{ccrrccc}
\hline & \multicolumn{3}{c}{ Relative deviation } & \multicolumn{2}{c}{ RF distance } \\
A & $\mathcal{F}_{\boldsymbol{\alpha}}(\mathcal{M})$ & \#adj & \#edg & \#exact results & exact & heuristic \\
\hline 0.001 & $-2.67 \%$ & $2.83 \%$ & $-0.23 \%$ & 43 & 2 & 2 \\
0.3 & $-3.47 \%$ & $0.90 \%$ & $0.31 \%$ & 63 & 2 & 2 \\
0.5 & $-4.26 \%$ & $-1.03 \%$ & $0.84 \%$ & 61 & 2 & 2 \\
0.8 & $-6.34 \%$ & $-1.71 \%$ & $1.14 \%$ & 54 & 4 & 2 \\
1 & $-8.41 \%$ & $-2.39 \%$ & $17.7 \%$ & 48 & 6 & 2 \\
\hline
\end{tabular}

Comparison of FFAdj-Int and FFAdj-MCS. The left side of the table shows the relative deviation in the objective function $\mathcal{F}_{\alpha}(\mathcal{M})$, the number of adjacencies (\#adj), and the size of the resulting matching (\#edg), of heuristic results from the exact solutions under varying values of $\alpha$. On the right, the RF distances between the true tree and trees based on exact and heuristic results are listed. both FFAdj-Int and FFAdj-MCS is on average larger than in Adjacencies-Intermediate-Matching.

\section{Evaluating phylogenies}

A good indicator for accuracy of a genome-based distance measure is the quality of the phylogenetic tree based on its drawn distances.

The distance measure that we used in this analysis resembles the breakpoint distance normalized by the size of the matching. For a given matching $\mathcal{M}$ of a size \#edg $(\mathcal{M})$ and a given number of adjacencies $\# \operatorname{adj}(\mathcal{M})$, the normalized number of breakpoints is $(\# \mathbf{e d g}(\mathcal{M})$ - \#adj $(\mathcal{M})-1) / \# \mathbf{e d g}(\mathcal{M})$. Now, since the objective of Problem FF-Adjacencies does not maximize adjacencies but rather a linear combination of $\operatorname{adj}(\mathcal{M})$ and $\operatorname{edg}(\mathcal{M})$, we define a distance measure based thereon:

$$
\Delta(\mathcal{M})=\frac{e d g(\mathcal{M})-\operatorname{adj}(\mathcal{M})-1}{e d g(\mathcal{M})}
$$

In our evaluation we applied the well-known Neighbor Joining Method (NJ) [18] for inferring phylogenetic trees. Subsequently we compared these to the tree proposed by Lerat et al. [14] that we assume to represent the true phylogeny. Thereby we used the Robinson Foulds topological distance (RF distance) [19] to evaluate our inferred phylogenetic trees. The results are shown on the right side of Table 2. For the majority of all cases we were able to reconstruct the tree correctly up to a single internal edge, causing an RF distance of 2 to the original tree. This internal edge connects the two organisms Buchnera aphidicola (BAPHI) and Salmonella typhimurium (SALTY) with the rest of the tree (Figure 4(a)). This branch is known to be particularly hard to reconstruct since the two organisms diverged far from each other, resulting in two long external edges in the tree. We also reconstructed the phylogeny based on 
gene families using the measures that were proposed in [13] and obtained an RF distance of 2 to the true tree under the intermediate model and an RF distance of 4 under the maximum matching model, featuring the same aberrancy. These results suggest that gene family information is not relevant in reconstructing the phylogeny of Lerat et al.'s tree. Yet, the increasing deviation from the true tree for the results of FFAdj-Int when $\alpha$ tends to 1 indicates that for genome comparison, the maximization of adjacencies is not enough. The fact that the heuristic outperforms the exact algorithm for $\alpha=1$ in terms of RF distance to the true tree confirms the importance of maximizing $\operatorname{edg}(\mathcal{M})$ as well. We recall that FFAdj-MCS iterates until complete saturation is obtained, which increases $\operatorname{edg}(\mathcal{M})$, even when $\alpha=1$.

Often, one cannot judge the tree-additivity of the underlying distances by investigating the fully resolved Neighbor Joining tree. Thus, in Figure 4 we provide a NeighborNet [20] representation of some of our obtained phylogenies. In the plots the internal edges that are hard to reconstruct are directly exposed, showing network-like rather than tree-like structures, in particular for the tree obtained from [13]. To conduct these phylogenetic analyses, we used the software packages PHYLIP [21] and SPLITSTREE [22].

\section{Conclusions}

In this work, we introduced the concept of comparative genomics by direct analysis of gene similarities without prior assignment of gene families. To illustrate this approach, we resorted specifically to one problem of gene order comparison: Finding a matching that identifies similarities between two genomes by maximizing conserved adjacencies and similarities for each pair of genes simultaneously. This problem is NP-hard. We propose to resolve it by an exact algorithm (efficient for small genomes) and a good heuristic. In our experiments on $12 \gamma$ proteobacterial genomes, we observed that the omission of gene families allowed for an increase in the number of adjacencies as well as the size of the matching while the resulting distances gain higher precision in reconstructing phylogenies.

Future work. This study is a preliminary work in a new field of comparative genomics wherein the assignment of gene family is unnecessary. Many studies can be explored. With regard to the specific problem studied here, our exact algorithm can be improved by rules which reduce the required main memory. Moreover, we believe that a hybrid heuristic - starting a pre-matching using the iterative heuristic until the size of the MCS is less than a parameter $k$, then finishing the matching with our exact

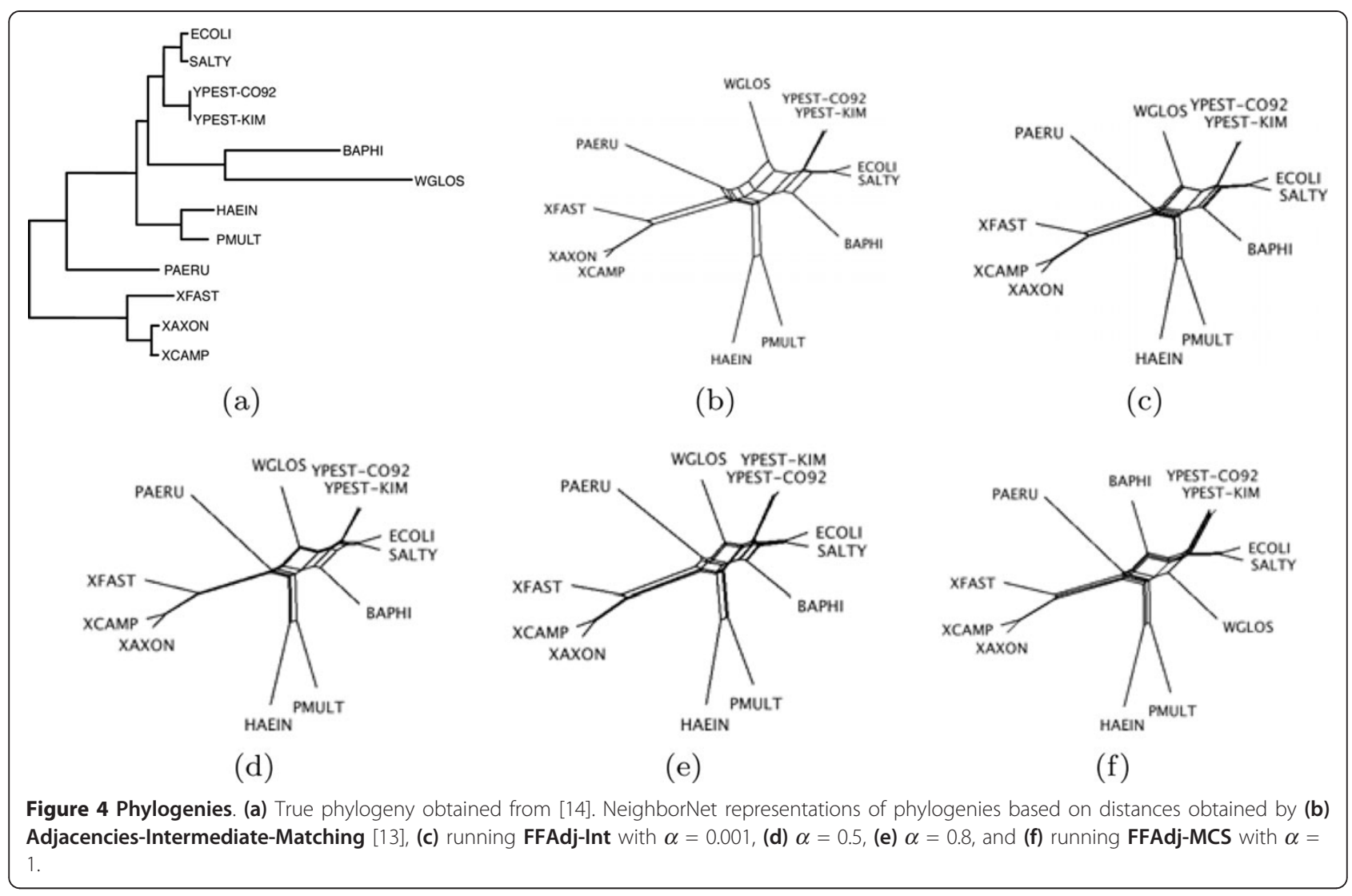


algorithm - can allow to find near-exact results for even larger genomes. On the other side, a deep study of the measure $\sigma$ can increase the quality of the comparison; comparing genes by sequence similarity is only one of many methods that can be applied.

From a more general point of view, this study shows that it is conceivable to extend the direct analysis approach to other types of gene order studies such as the computation of DCJ distances or gene cluster prediction.

\section{Additional material}

Additional file 1: Measured adjacencies between $12 \gamma$-proteobacterial genomes. Values obtained from FFAdj-Int and FFAdj-MCS. \#adj denotes the number of conserved adjacencies in the matching and \#edg indicates the number of its edges. $X$ indicates that the exact calculation did not terminate due to the lack of sufficient memory.

\section{Acknowledgements}

DD receives a scholarship from the CLIB Graduate Cluster Industrial Biotechnology. AT is a research fellow of the Alexander von Humboldt Foundation.

This article has been published as part of BMC Bioinformatics Volume 13 Supplement 19, 2012: Proceedings of the Tenth Annual Research in Computational Molecular Biology (RECOMB) Satellite Workshop on Comparative Genomics. The full contents of the supplement are available online at http://www.biomedcentral.com/bmcbioinformatics/supplements/ 13/S19.

\section{Author details}

'Genome Informatics, Faculty of Technology, Center for Biotechnology (CeBiTec), Bielefeld University, Germany. ${ }^{2}$ Institute for Bioinformatics, Center for Biotechnology (CeBiTec), Bielefeld University, Germany.

\section{Authors' contributions}

All authors participated in discussing, formulating, and conducting the research. Also, all authors contributed to the writing and editing of the manuscript and read and approved the final manuscript.

\section{Competing interests}

The authors declare that they have no competing interests.

Published: 19 December 2012

\section{References}

1. Paten B, Earl D, Nguyen N, Diekhans M, Zerbino D, Haussler D: Algorithms for genome multiple sequence alignment. Cactus Genome Research 2011, 21(9):1512-1528

2. Tatusov RL, Fedorova ND, Jackson JD, Jacobs AR, Kiryutin B, Koonin EV, Krylov DM, Mazumder R, Mekhedov SL, Nikolskaya AN, Rao BS, Smirnov S, Sverdlov AV, Vasudevan S, Wolf YI, Yin JJ, Natale DA: The COG database: an updated version includes eukaryotes. BMC Bioinformatics 2003, 4:41.

3. Powell S, Szklarczyk D, Trachana K, Roth A, Kuhn M, Muller J, Arnold R, Rattei T, Letunic I, Doerks T, Jensen $\sqcup$, von Mering C, Bork P: eggNOG v3.0: orthologous groups covering 1133 organisms at 41 different taxonomic ranges. Nucleic Acids Res 2011, 40(D1):D284-D289.

4. Remm M, Storm CE, Sonnhammer EL: Automatic clustering of orthologs and in-paralogs from pairwise species comparisons. J Mol Biol 2001, 314(5):1041-1052.

5. Li H: TreeFam: a curated database of phylogenetic trees of animal gene families. Nucleic acids res 2006, 34(90001):D572-D580.
6. Chiu JC, Lee EK, Egan MG, Sarkar IN, Coruzzi GM, DeSalle R: OrthologID: automation of genome-scale ortholog identification within a parsimony framework. Bioinformatics 2006, 22(6):699-707.

7. Fu Z, Jiang T: Clustering of main orthologs for multiple genomes. J Bioinform Comput Biol 2007, 6:195-201.

8. Watterson G, Ewens W, Hall T, Morgan A: The Chromosome Inversion Problem. J Theor Biol 1982, 99:1-7.

9. Stoye J: Computation of Median Gene Clusters. J Comput Biol 2009, 16(8):1085-1099.

10. Yancopoulos S, Attie O, Friedberg R: Efficient sorting of genomic permutations by translocation, inversion and block interchange. Bioinformatics 2005, 21(16):3340-3346.

11. Bergeron A, Mixtacki J, Stoye J: A unifying view of genome rearrangements. Proc of WABI 2006, 163-173.

12. Tang J, Moret BME: Phylogenetic Reconstruction from GeneRearrangement Data with Unequal Gene Content. Proc of WADS 2003, $37-46$

13. Angibaud S, Fertin G, Rusu I, Thévenin A, Vialette S: Efficient tools for computing the number of breakpoints and the number of adjacencies between two genomes with duplicate genes. J Comput Biol 2008, 15(8):1093-1115

14. Lerat E, Daubin V, Moran NA: From Gene Trees to Organismal Phylogeny in Prokaryotes:The Case of the $\gamma$-Proteobacteria. PLoS Biology 2003, 1:e9.

15. Blin G, Chauve C, Fertin G: Genes Order and Phylogenetic Reconstruction: Application to $Y$-Proteobacteria. Proc of RECOMB-CG 2005, 11-20.

16. Williams KP, Gillespie JJ, Sobral BWS, Nordberg EK, Snyder EE, Shallom JM, Dickerman AW: Phylogeny of Gammaproteobacteria. J Bacteriol 2010, 192(9):2305-2314.

17. Pesquita C, Faria D, Bastos $H$, Ferreira $A E$, Falcão $A O$, Couto FM: Metrics for GO based protein semantic similarity: a systematic evaluation. BMC Bioinformatics 2008, 9(Suppl 5):S4.

18. Saitou N, Nei M: The neighbor-joining method: a new method for reconstructing phylogenetic trees. Mol Biol Evol 1987, 4(4):406-425.

19. Robinson D, Foulds L: Comparison of Phylogenetic Trees. Math Biosci 1981, 53:131-147.

20. Bryant D: Neighbor-Net: An Agglomerative Method for the Construction of Phylogenetic Networks. Mol Biol Evol 2003, 21(2):255-265.

21. Felsenstein J: PHYLIP-Phylogeny Inference Package (Version 3.2). Cladistics 1989, 5:164-166.

22. Huson DH: Application of Phylogenetic Networks in Evolutionary Studies. Mol Biol Evol 2005, 23(2):254-267.

\section{doi:10.1186/1471-2105-13-S19-S3}

Cite this article as: Doerr et al:: Gene family assignment-free comparative genomics. BMC Bioinformatics 2012 13(Suppl 19):S3.

\section{Submit your next manuscript to BioMed Central and take full advantage of:}

- Convenient online submission

- Thorough peer review

- No space constraints or color figure charges

- Immediate publication on acceptance

- Inclusion in PubMed, CAS, Scopus and Google Scholar

- Research which is freely available for redistribution

Submit your manuscript at www.biomedcentral.com/submit
Ciomed Central 